\title{
Formalizing and Modeling Enterprise Architecture (EA) Principles with Goal-oriented Requirements Language (GRL)
}

\author{
Diana Marosin ${ }^{1,2}$, Marc van Zee ${ }^{3}$, Sepideh Ghanavati ${ }^{2}$ \\ 1 Luxembourg Institute of Science and Technology (LIST), Luxembourg \\ 2 Radboud University Nijmegen, the Netherlands \\ 3 University of Luxembourg, Luxembourg \\ diana.marosin@list.lu, marc.vanzee@uni.lu, s.ghanavati@cs.ru.nl
}

\begin{abstract}
Enterprise Architecture (EA) principles are normally written in natural language which makes them informal, hard to evaluate and complicates tracing them to the actual goals of the organization. In this paper, we present a set of requirements for improving the clarity of definitions and develop a framework to formalize EA principles with a semi-formal language, namely the Goal-oriented Requirements Language (GRL). We introduce an extension of the language with the required constructs and establish modeling rules and constraints. This allows us to automatically reason about the soundness, completeness and consistency of a set of EA principles. We demonstrate our methodology with a case study from a governmental organization. Moreover, we extend an Eclipse-based tool.
\end{abstract}

Keywords: enterprise architecture principles, Goal-oriented Requirements Language, OCL rules, formalism, analysis

\section{Introduction}

In practice, Enterprise Architecture (EA) is driven top-down by the business and/or IT strategies and bottom-up by projects and programs which are often owned and driven by the business units. These initiatives, programs and projects are often evaluated via EA principles. EA principles aim to ensure that current and new projects and programs are aligned with, and not deviating from, the business and IT strategies $[26,18,22]$. In this context, EA principles refer to either the engineering view on the EA (such as how the elements of the EA must be implemented or changed to provide a certain functionality and what business requirements are needed for this functionality), or the social view (such as the ones related to organization's culture or human aspects). For example, an EA principle "Be friendly with the client" can refer to both the social and engineering views. The social view of this principle could mean that the organization recommends its employees to be nice to the customers, which is an informal recommendation and cannot be enforced. Whereas, the engineering view of this principle could mean that the organization has a good IT infrastructure and intends to develop a user-friendly software experience. This latter engineering view 
can be formalized and enforced. In our work, we aim to focus on the engineering view of the EA principles that can be modeled, formalized and enforced.

Based on the experience with our industry partner, EA principles are usually written informally in a natural language format. This can cause ambiguities and lead to several interpretations. Moreover, there is no mechanism for consistency checking between various EA principles. In addition, the traceability between EA principles and the goals, objectives and strategies of the organizations are not fully documented. This lack of traceability can become problematic, particularly when the organizations' objectives and strategies change or a new project is introduced to it. It can also lead to difficulties in analyzing the impact of EA principles on the goals and objectives of organizations, as well as difficulties in performing trade-off analysis deciding to which EA principles adhere to.

A methodology for formal representation of EA principles together with a tool support can help organizations reduce the issues presented above. In recent years, much work has been done in other research fields such as regulatory compliance and goal-oriented requirements engineering in formalizing regulations and business rules (cf. Nómos3 [16] and LEGAL-URN [9] for analyzing regulatory compliance between laws, regulations and business rules and processes.) Furthermore, there are open-source tools such as jUCMNav [3] which support the representation and analysis of goal-oriented models. These approaches have shown that having a structured framework with tool-support can ensure that the organization's goals are aligned and in compliance with the current regulations and laws. Similarly, we look at EA principles from an engineering and normative perspective, noting that EA principles share similar characteristics with softlaws [20]: semi-structured, represented in natural language and informal. Thus, a goal-oriented modeling approach could be suitable for formalizing, modeling and analyzing EA principles [21].

The identified issues with EA principles' formalism, as well as their resemblance with soft-laws and the previous work conducted in formalizing laws and business processes and analyzing of regulatory compliance, lead us to the following research question:

How can we represent EA principles in a semi-formal structure with a goal-oriented modeling language? What are the needed language constructs? What are the modeling constraints?

We select Goal-oriented Requirements Language (GRL) [2] as the modeling language for formalizing EA principles. GRL is part of the User Requirements Notation (URN) [17] which is currently the only modeling language part of the ITU-T standard. Furthermore, URN has an open-source, Eclipse-based toolsupport, called jUCMNav [3]. GRL supports different bottom-up and top-down evaluation mechanisms (e.g., qualitative, quantitative, hybrid) that allow analysis of goal satisfaction and trade-off analysis when choosing between different alternatives. New profiles can be created by annotating GRL intentional elements with stereotypes and incorporating constraint rules written in Object Constraint Language (OCL). The language can also be extended with the help of Metadata and URN links. To that end, we use and extend the jUCMNav plug-in with a principle-base set of stereotypes and OCL rules. 
To answer our research question, we apply a design science methodology [30, 14] driven by practice. In our previous work, we analyzed the EA principles of the Schiphol group [21] and then mapped them to GRL intentional elements. In the current iteration, we conducted a more extensive literature study building on the work of Stezler [25] and Haki [12], analyzing the existing definitions of EA principles. In this paper, we provide guidelines on how to define EA principles in a more structured format and how to model them with GRL. We aim to improve the Principle-based GRL profile to capture various principle structures. We provide steps for modeling and we define means to verify the correctness and well-formedness of the GRL models. We validate our approach with a new case study within a governmental organization in Europe. We uploaded our GRL extension, together with the case study description, the resulted models and instructions to an online repository ${ }^{4}$.

Following our methodology, we first introduce the related work in Sect. 2. We present the steps for modeling EA principles with GRL in Sect. 3. We evaluate our approach with a case study and present the case results in Sect. 4. We conclude and present directions for future work in Sect. 5 .

\section{Related Work}

In EA, principles have been defined as guidelines and rationales for the design and evolution of technology plans $[15,26,24,4,10]$. In other words, EA principles can be seen as "rules of conduct" and can be made more precise and operational by formalization. The empirical studies conducted by Haki, Fischer and Winter (cf. $[32,8,12,13]$ ) provide insights from a practitioners' point of view on the use of principles. According to them, one of the difficulties in the use and adoption of EA principles is caused by a lack of understanding of their impact. According to Winter [32], EA principles miss a methodology for the adoption and application guidelines and there are no regular checks for usefulness and consistency.

Efforts in formalizing EA principles were made by Chorus et al. [6] and Bommel et al. $[28,27]$. The authors present a collection of EA principles from TOGAF and show the feasibility of formalization. The approach is done in two steps. The first step consists of interpreting the EA principles as defined in natural language. The authors identified a set of issues regarding the interpretation from natural language. The second step includes the representation of EA principles as an ORM/ORC expression ${ }^{5}$. Their approach has limitations since the EA principles are represented in isolation. They are not connected to either the goals of the organization or other EA principles. Therefore this formalism does not support the impact analysis. Platianiotis et al. [23] proposed a conceptual framework for tracing the EA design decisions to their rationals, however the method does not provide any formalism for the EA principles. Additionally, the framework proposed by van Zee et al. [29] contains logic-based rules for verifying the integrity of EA decisions, but does not consider EA principles.

\footnotetext{
${ }^{4}$ https://github.com/RationalArchitecture/eGovernment

${ }^{5}$ Object Role Modeling and Object-Role Calculus
} 
ARMOR [7], which is based on Goal-Oriented Requirements Engineering (GORE) approach aims to fill the gap for modeling motivation but it does not focus on formally modeling the principles. Moreover, ARMOR is not scalable and does not include automatic analysis mechanism and tool support. On the other hand, the motivational extension to ArchiMate [5] tries to help representing EA principles in terms of goals and rationales, but does not provide any formalism.

Focused on information systems, Akhigbe et al. [1] introduce an adaptive EA framework (BI-EAEA ${ }^{6}$ ), that allows one to pro-actively accommodate the changes that occur in evolving settings (e.g., how an enterprise responds to various changes, such as modification, deletion and addition of organizations' objectives). However, this method does not provide any formalization for EA principles and it only evaluates changes without considering the EA principles' impacts.

We previously introduced a framework called Principles-based GRL [21], in which we made the first steps towards formalizing EA principles and set grounds on checking compliance between EA principles and EA models [20]. In this paper, we aim to improve the GRL profile for principles and introduce constraints for modeling EA principles (i.e. OCL rules). Our approach aims to represent EA principles in terms of rationals, goals and operational actions. It also takes advantage of the GRL tool-support provided by using jUCMNav. jUCMNav allows users to break the model into hundreds of diagrams and intentional elements and perform GRL analysis for all of the models. This feature which helps scalability of GRL also solves the scalability issues mentioned in the literature.

\section{Goal-oriented based Framework for EA Principles}

In this section we aim to:

1. state the requirements for defining a set of EA principles

2. create a consistent definition of EA principles that enable us to analyze the EA principles in the Goal-oriented Requirements Language (GRL).

3. define modeling constraints (i.e. OCL rules) for ensuring the well-formedness of EA principles when represented in GRL.

\subsection{Requirements for Defining a Set of EA Principles}

Lindstrom [19] uses the characteristics of "good requirements, originating from requirements engineering" (e.g., IEEE Std 830-1998, Software Requirement Specification) to define the requirements for a good set of EA principles. The authors distinguish between syntax (the form of the principle) and semantics (the meaning and content of the principles). The criteria for assessing the quality of EA principles are as follows: verifiability, completeness, correctness, modifiability, unambiguity, consistency and stability. Similarly, TOGAF [26] lists five criteria that distinguish a set of good EA principles: understandable, robust, complete, consistent and stable.

\footnotetext{
${ }^{6}$ Business Intelligence - Enabled Adaptive Enterprise Architecture
} 
Op 't Land and Proper [22] define two methodologies on how to create a SMART set of EA principles (e.g., specific, measurable, achievable, realistic, time-related). The authors introduce the notion of prioritizing the EA principles based on the key objectives (contrary to Lindstrom who intentionally left out these issues.) An important requirement is the completeness of the set of EA principles.

Based on the requirements given by TOGAF and Op 't Land, we define the following requirements for a set of EA principles.

1. Understandable: Each principle should be sufficiently definitive and precise to be quickly grasped and understood by individuals and to support consistent decision making in complex, potentially controversial situations. This definition is a result of combining the properties Unambiguous, Robust and Specific.

2. Complete: Every potentially important principle governing the management of the organization is defined. We intentionally left out the reference to IT and technology as defined in TOGAF [26] and created a more general requirement. Lindstrom [19] states that when validating if the principles are correct and complete the following questions must be asked: "Are the stated principles relevant to the organization?", "Are all necessary principles defined?"

3. Consistent: Principles should not be contradictory to the point where adhering to one principle would violate the goal of the other. Note that in practice, we have found conflicting EA principles. Multiple violations of one or another principle can result in a revision of the set of EA principles, until the set becomes consistent [21].

4. Measurable: Both on long-term and short-term, over the future architecture and project portfolio. Measurements are needed to assure that the organization's goals are achieved and to check if the EA principles are really followed and what is their impact on the organization [19].

5. Stable: Principles should be enduring, yet able to accommodate change. There is a need to establish a methodology for changing the set of principles and this should be triggered when a) a strategy or goals of the organization change; b) principles are conflicting; c) principles are constantly violated.

\subsection{Mapping EA Principles to Goal-oriented Requirements Language Constructs}

In this subsection, we

- summarize the definitions of EA principles as found both in the current academic literature and practice

- introduce the GRL constructs and the stereotypes needed to formalize the EA principles

- we present a mapping of EA principles to GRL constructs

Goal-oriented Requirements Language (GRL), which is based on the $i^{*}$ language, describes business concerns, goals satisfactions and stakeholders' be- 
liefs and dependencies. It can be extended and become domain specific by using stereotypes attached to the basic constructs of the language. Introducing stereotypes allows us to first define a domain specific notation for the EA principles and then introduce restrictions for the modeling language to assure the well-formedness of the models.

We summarize the definitions of the existing constructive elements of EA principles as found both in the current academic literature and practice, and we annotate them with stereotypes as presented in Table 1. All stereotypes related to EA principle are grouped under the name ST_Principle. An example of the mapping is shown in Fig. 3.

Table 1: Mapping EA Principles Constructs to GRL Intentional Elements \begin{tabular}{|l|l|l|}
\hline EA Principle Element & Stereotype Value & GRL Element
\end{tabular}

\begin{tabular}{|c|c|c|}
\hline Name & $\ll$ Principle $\gg$ & Softgoal (@) \\
\hline Statement & - & Comment \\
\hline Added Value & $\ll$ AddedValue $\gg$ & Softgoal, Goal $(\bigcirc)$ \\
\hline Impact/restrictions & 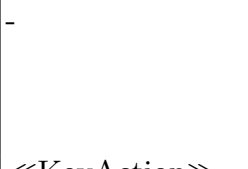 & $\begin{array}{l}\text { The value of GRL links (e.g., quantitative im- } \\
\text { pact (integer value between - } 100 \text { and } 100 \text { ) or } \\
\text { a qualitative value, marked with the keywords } \\
\{\text { make, help, some+, some-, hurt, break }\} \text {. }\end{array}$ \\
\hline Key Actions & $\ll$ KeyAction $\gg$ & Task $(\triangleright)$ \\
\hline Preconditions & $\ll$ Precondition $\gg$ & Softgoal, Goal, Task, Resource ( $\square)$ \\
\hline Architecture Domain & - & Actor $\left(e^{-}\right)$ \\
\hline
\end{tabular}

- Name: This field captures the essence of the principle and should be easy to remember $[22,26,11]$. EA principles are not part of the GRL language definition. In order to evaluate the impact of the EA principles on a goal (and vice versa), we introduce an element that explicitly refers to the EA principle itself and stereotyped it with the annotation «Principle» IE. For example, Principle 4 (We prefer to communicate digitally with citizens and businesses of Sect. 4 is annotated by «Principle» softgoal in GRL in Fig. 3.

- Statement: This field is a clear, unambiguous description of the principle $[22,26,11]$. The statement is in some sense a summary of the EA principle, very useful in human communication, but does not necessarily carry much semantics in a formal language. Therefore, it is stored as a comment that is attached to the EA «Principle» IE.

- Added value: This field states clearly what is aimed to be achieved when applying the EA principle (e.g., goals/softgoals to which the principle contributes to, either positively or negatively). Different researchers have named this field differently such as Motivation [22,31] or Rational [26, 8]. We found it in practice ${ }^{7}[21]$ also under the names Future situation and Goal. We represent the added value in GRL as a softgoal or a goal, to which we attach

\footnotetext{
${ }^{7}$ Schiphol airport case study
} 
«AddedValue» stereotype. In Fig. 3, Fast and efficient communication is one of the «AddedValue» of Principle 4.

- Impact and Restrictions: This field defines the impact of an EA principle on the design of other principles or elements of the architecture, as well as the restrictions caused by enforcing the principles [8]. In practice [21], it is also called Constraints. It can also be called Implications $[22,24,19]$. We chose to use the term Impact, as it is less ambiguous in this context. This is modeled in GRL by a contribution/correlation link (both with positive or negative values). Contribution links show the direct impact of one intentional element to the other while correlation links show the side-effect. Both links can have qualitative or values. The links between «Principle» and other intentional elements in Fig. 3 represent impact.

- Key actions: This field states what the operational actions to be taken are so that the principle is realized. In practice [21], it is also called Application, Key Actions [8], Assurance [22] or Implications [15]. This element corresponds to task in GRL and it is annotated by «KeyAction》stereotype. We offer supplementary possibilities to be contacted is a $\ll$ KeyAction》 in Fig. 3.

- Preconditions: This field contains preconditions and requirements to be fulfilled before the principle can be applied. In practice, we found this field under the name Implications ${ }^{8}$. Hoogervorst [15] introduces the field key actions for effectuating the architecture to ensure that the principle can be followed. We introduce a new element in GRL «Precondition》 IE. A precondition can be modeled as a task, resource, softgoal or goal. An example of a $\ll$ Precondition》 is Clear view of the customer.

- Architecture domain: This field states to which part of the architecture the principle is applied (e.g., business, infrastructure, organization...) $[15,4$, $21]$. We use the concept of "actor" for bounding in representation of the GRL intentional elements that constitute an EA principle. EA Principle 4 is an actor in Fig. 3 which includes Principle 4 and all of its intentional elements.

\subsection{OCL Rules for Checking the Well-Formedness of EA Principles}

The Object Constraint Language (OCL) is a declarative language for describing rules that apply to formal models. Due to its Eclipse OCL plug-ins that support rule definitions, checking, and explanation, OCL can be integrated with jUCMNav. It is, therefore, possible to define and verify OCL rules for any GRL model. We provide ten OCL rules for checking the well-formedness of the EA principles. Given the space restrictions for this paper, we exemplify in Fig. 1 the implementation of one OCL rule ${ }^{9}$.

1. An EA principle must be modeled as a softgoal. (PrincipleAsSoftGoal) As stated before, the EA principles are seen as "rules of conduct"

\footnotetext{
8 eGovernmnet case study

9 The implementation of the rest of OCL rules is part of the Principle-based GRL framework and can be found at https://github.com/RationalArchitecture/ eGovernment
} 
and cannot be fully enforced. Since the EA principles are high-level and somewhat vague in nature, we enforce modeling any element that has the stereotype «Principle» as a softgoal intentional element.

2. A key action must be modeled as a task. (KeyActionAsTask) By refining the EA principles to the level of tasks we ensure we operationalize the usage of EA principles.

3. The added value must be modeled as a softgoal or as a goal. (AddedValueAsGoalOrSoftgoal) EA principles should have an impact on the high level goals or softgoals of the organization. Softgoals, represent what a stakeholder wants to achieve. Contrary to goals, softgoals do not have quantifiable measurements. Goals, however, are more precise, have quantifiable measurements and can be clearly achieved.

4. EA Principles, added values, preconditions, and key actions cannot be modeled as beliefs. (BeliefsNotStereoTyped) This rule is required since beliefs in GRL are different entities from the intentional elements (i.e. goals, softgoals, resources, and tasks). Beliefs capture the rationales and justifications of GRL intentional elements and their links.

5. Each EA principle must have at least one contribution from a key action. (KeyActionToPrinciple) In order to operationalize the EA principles, we consider it necessary to refine their definition until we reach the tasks' level. This means that it is necessary to clearly define the key actions for realizing the EA principle. Therefore, each intentional element that has the stereotype 《Principle» must be refined and have at least one contribution from an intentional element with the stereotype $\ll$ KeyAction».

6. If a precondition is introduced using a contribution link, the link must get the maximum value. (ContributionFromPreconditionIsMax) In GRL, the evaluation algorithms depend on the values of the links. By giving the contribution the maximum value (e.g., 100 or make), we enforce that the precondition has at least high priority in the evaluation as the other intentional elements linked to the parent.

7. If a precondition is introduced using a dependency link, the precondition must be modeled as source. (PreconditionAsSourceOfDependency) In GRL notation, the dependency links are modeled as follows: target $\rightarrow-$ source. We introduce this OCL rule in order to assure that preconditions are modeled correctly in GRL notation. A dependency link shows a relationship between a dependent intentional element which depends on a precondition intentional element. At the time of the evaluation, the intentional element dependent on a precondition receives the minimum value between its own evaluation and the evaluation of the precondition.

8. Each EA principle must contribute to at least one (soft)goal (here stereotyped «AddedValue») of the organization. (PrincipleToGoal) By introducing this rule we assure that we do not introduce an EA principle that has no real value for the goals of the organization.

9. Each (soft)goal (here stereotyped «AddedValue $\gg$ ) of the organization must have at least one contribution link from the set of EA prin- 
ciples. (GoalToPrinciple) By introducing this rule, we assure that every goal of the organization is also addressed by at least one EA principle.

10. The EA principles should not propagate a "conflict" satisfaction value for added value. (NoConflicts) A set of two or more EA principles must not have contradictory contribution links on the same goal. If this happens and the goal gets "conflict" satisfaction value, a warning is triggered and the set of EA principles has to be revised in such a way that it is kept consistent.

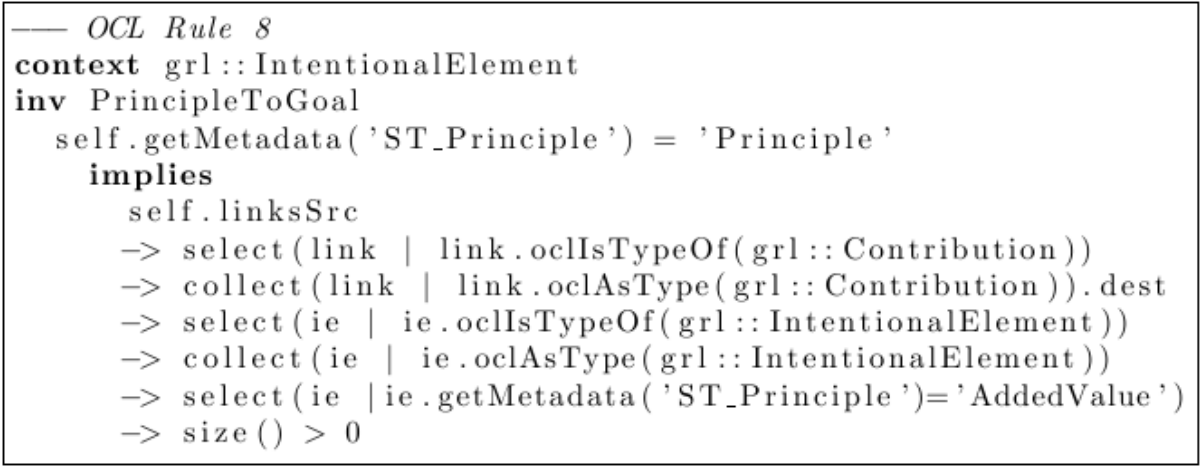

Fig. 1: Implementation of OCL Rule 8: PrincipleToGoal

\subsection{Synthesis}

In Sect. 3.1, we presented requirements for a set of good principles based on a summary of previous approaches found in the literature (e.g. [26, 19, 22]). Furthermore, in Sect. 3.2, we revised definitions of EA principles from literature (e.g. $[15,18,26,31,32])$ and practice (e.g. [21]) and created a unified structure for EA principles. We mapped these constitutive elements of EA principles to constitutive elements of GRL, creating a Principle-based GRL profile. In order to check the correctness of the GRL models for EA principles we defined ten OCL rules in Sect. 3.3. In Table 2, we check the properties of a good set of principles over the defined OCL rules.

\section{Evaluation}

In the previous sections, we presented our motivation for formalizing EA principles in GRL, along with the steps and requirements for this task. We evaluate our approach with a case study. We offer a posteriori rationalization and formalization of the EA principles used by a governmental organization whom for professional reasons wishes to remain anonymous, hereafter called eGovernment. 
Table 2: Verifying the Requirements for a Good Set of EA Principles over the EA Principles' OCL Rules

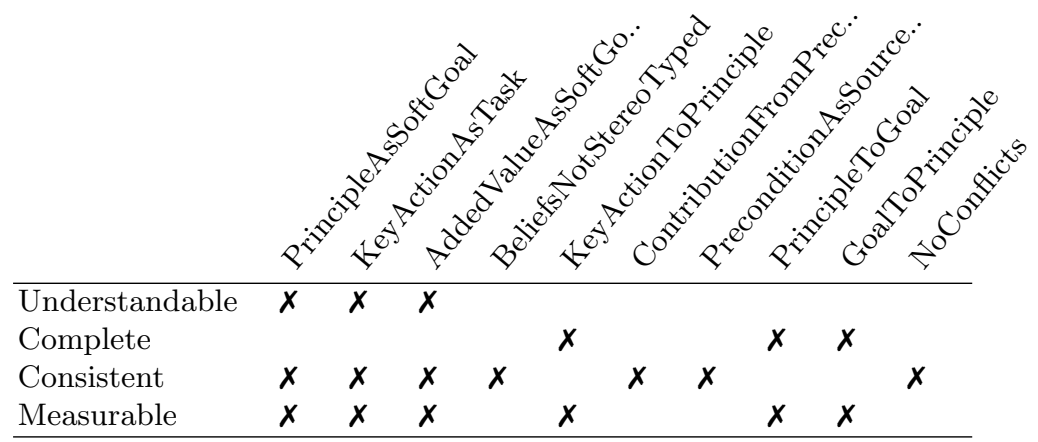

\subsection{Case Study Presentation}

eGovernment is the tax collection and customs administration of a European country. As part of the Ministry of Finance, eGovernment is responsible for supervising the import, export and transit of goods, detecting fiscal, economic and financial frauds, levying and collecting taxes, and paying out income-related benefits for child care, rent and health care.

The administration of eGovernment has discussed about how they envision their organization in 2020: an efficient governmental organization that achieves maximum compliance at minimum cost. Furthermore, an organization that is well in contact with citizens and businesses, interacts with them and adapts to their needs and behaviors. Changing circumstances, such as aging population, globalization and digitalization require eGovernment to transform itself. This emerging vision and the way eGovernment wants to be positioned into society was translated into nine design principles to guide their transformation.

We choose to analyze in detail a particular EA principle regarding the digitalization of eGovernment, as presented in Fig. 2.

\subsection{Results: Formalization and Modeling of EA principles in GRL}

In this section, we first provide the extended definition of eGovernment's EA Principle: We prefer to communicate digitally with citizens and businesses. Next, we analyze and interpret the statements and create the GRL model for the principle based on the mapping and OCL rules of Sect. 3. This definition has been translated from its original language to English. We tried to avoid any interpretation errors and kept the translation as close to the original meaning as possible.

Originally the EA principles of eGovernment are represented in natural language using an adaptation of the EA principles' structure from TOGAF [26]: name, statement, rationale and implications. In this context, the term "Rationale" refers to the goals that are tackled by applying the principle and "Implica- 
Principle 4: We prefer to communicate digitally with citizens and businesses (1) Supplementary possibilities to contact us are only offered when these have additional values in the communication. If that is too much of the case, then we ensure that citizens and companies receive consistent information despite the channel from which they are served. (2)

- Digital communication is fast and efficient for both parties. (3)

- Since we aim for compliant behavior and due to the fact that digital communication does not contribute to that in all cases, we offer supplementary possibilities to contact us. (4)

- Since we are a governmental organization, we are obliged to make our services accessible to everybody, digitally experienced or not. (5)

This means that:

- We persuade citizens and companies to use Internet, the preferred medium. (6)

- We realize consistency in policy and execution over the different channels. (7)

- Information and transactions of citizens and companies is processed via different channels into a clear view of the customer. (8)

- We offer all electronic transaction services via two channels: a basic service via a portal and a system-to-system matching (as much as possible using TechnologyX via Portal) for the fiscal employees and entrepreneurs who use commercial software packages. (9)

Fig. 2: Original Definition and Translation of eGovernment's EA Principle: We prefer to communicate digitally with citizens and businesses

tions" refers to the requirements and preconditions that have to be fulfilled. In Fig. 2, the name is represented in bold text and the statement is composed of the following paragraph. The first set of bullet points represent the "Rationale" and those under "That means that" represent the "Implications". We observed that the natural language text yields additional information about the Preconditions and Key Actions for operationalization of the EA principle.

We reorganize the original representation of the EA principle in such a way that it follows the EA principles' definition presented in Sect. 3. We identified the following information in a tabular format: natural language description, NL Statement, (for which we marked in Fig. 2 each statement), the simplified GRL IE description, GRL Desc., the reference of the current GRL element, Ref., the type of the GRL intentional element and the stereotype when applicable, GRL Type, the element to which it is related, Linked to, the relation type, Rel. Type, and the link value, Link Val.. Additional information, such as the actor or comments regarding the modeling solution or difficulties in interpretation of the natural language description can also be included. We present a partial semi-formal tabular representation of eGovernment's EA principle: We prefer to communicate digitally with citizens and businesses in Table 3.

One limitation for the a posteriori rationalization and the formalism is the challenge of evaluating the contribution links. In this example, we have introduced the values randomly. This step should be performed together with the 
architects and other involved stakeholders. A second challenge is raised by the interpretation of the natural language statements.

Table 3: Partial Representation of eGovernment's EA Principle 4 in a semiformal definition

\begin{tabular}{|l|l|l|l|l|l|l|}
\hline $\begin{array}{l}\text { Natural lan- } \\
\text { guage state- } \\
\text { ment }\end{array}$ & $\begin{array}{l}\text { GRL descrip- } \\
\text { tion }\end{array}$ & Ref. & GRL IE Type & $\begin{array}{l}\text { Linked } \\
\text { to }\end{array}$ & Rel. Type & Link value \\
\hline $\begin{array}{l}\text { (1) We prefer } \\
\text { to } . .\end{array}$ & $\begin{array}{l}\text { We prefer } \\
\text { to communi- } \\
\text { cate... }\end{array}$ & EAP4 & $\begin{array}{l}\text { Softgoal } \\
\ll \text { Principle }\end{array}$ & AV1 & Contrib & Help \\
\hline $\begin{array}{l}\text { (3) Digital } \\
\text { communica- } \\
\text { tion is... }\end{array}$ & $\begin{array}{l}\text { Fast and effi- } \\
\text { cient commu- } \\
\text { nication }\end{array}$ & AV1 & $\begin{array}{l}\text { Softgoal } \\
\ll \text { AddedValue } \gg\end{array}$ & EAP4 & & \\
\hline $\begin{array}{l}\text { (5) Since we } \\
\text { are a govern- } \\
\text { mental.. }\end{array}$ & $\begin{array}{l}\text { Make our ser- } \\
\text { vices accessi- } \\
\text { ble.. }\end{array}$ & $\begin{array}{l}\text { Task } \\
\ll \text { KeyAction } \gg\end{array}$ & EAP4 & Contrib. & Some+ \\
\hline $\begin{array}{l}\text { (7) We realize } \\
\text { consistency } \\
\text { over.. }\end{array}$ & $\begin{array}{l}\text { We realize } \\
\text { consistency } \\
\ldots\end{array}$ & Task & KA1 & Contrib. & Make \\
\hline
\end{tabular}

Abbreviations: $E A P=$ Enterprise Architecture Principle; $A V$ = AddedValue; $K A=$ KeyAction

\subsection{Analysis of the EA Principles and GRL Models}

In eGovernment, the EA principles (also originally called Appointments) were introduced with social aspects of the organization in mind. The EA principles were left ambiguous so that they could raise questions and bring people together for negotiations and finding solutions.

In our analysis, we identified and could confirm the following facts: EA principles are ambiguous. They have no traceability links to strategies, other principles, or activities of the organization. EA principles do not have a change management mechanism in place and have no formal mechanism for checking compliance. We also noticed that our formalism is more appropriate to the technology related EA principles. That is why we chose the EA principles related to technology, applications and infrastructure to formalize.

\subsection{Results: Evaluation from eGovernment's Enterprise Architects}

To ensure GRL models captured the essence of the EA principles correctly, we evaluated the models with two Enterprise Architects from eGovernment. The architects found the GRL models very useful in that they could visualize the principles and were easier for them to understand the links between the rationale, 


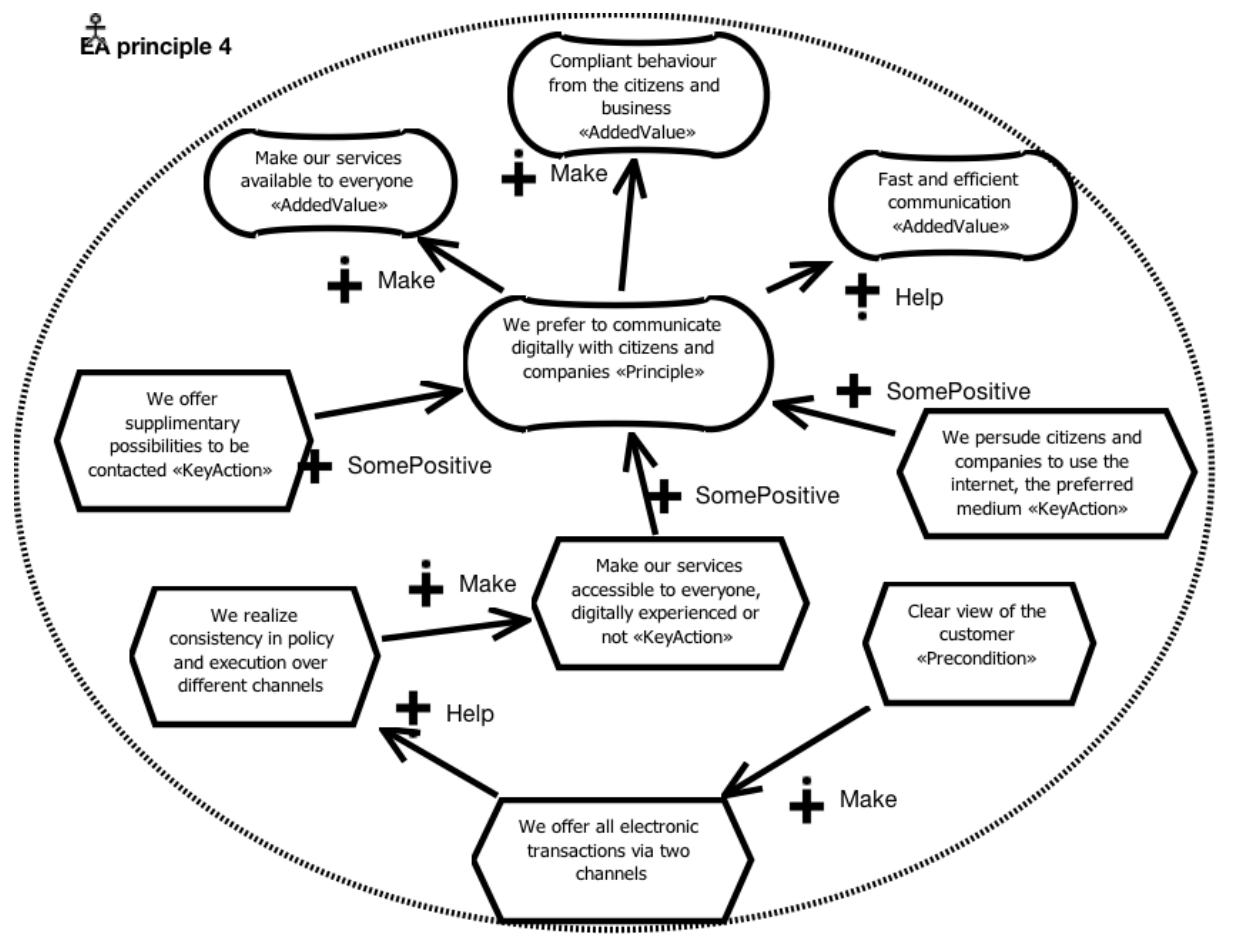

Fig. 3: GRL Representation of eGovernment's EA Principle 4 
added value, actions and the goals of the principles. Furthermore, since the EA principles were linked to the high-level goals of the organization, it made them easier to justify and analyze. Also, we captured the traceability links between the related EA principles which helped the architects to document the links and verify the connections. The architects also informed us that assigning values for contribution links can be done at design time together with the architects.

We aim to extend our models to more principles and provide analysis of the principles with high-level goals as well as low-level operationalized activities of the organization.

\section{Conclusions and Future Work}

In this paper, we focused on how to formalize and represent EA principles in a goal oriented modeling-language. For this, we first presented five requirements for good sets of EA principles and how to create EA principles. We, then, introduced a Principle-based GRL profile by adding stereotypes to the intentional elements of GRL. The correctness of the models is assured by defining ten OCL rules. Our method was applied to a case study from a European governmental organization. We formalized their technology related EA principles in GRL and conducted interviews with the involved architects for evaluation.

In future work, we will focus on creating a formal change mechanism for revising the set of EA principles. This is a missing item for creating a "stable" set of EA principles. First, changes in the set of principles (e.g., addition, deletion or modification of principles) trigger consistency checks on the models and revision of the current landscape, second, analysis of the current situation (e.g., current objectives or current environment of the organization, as well as addition of new projects and programs) trigger changes in the set of principles, potentially making the principles outdated and create conflicts with the new goals, new situations and potential imposed regulations. In order to realize the second type of revision, good traceability links between EA principles and business processes and architecture are needed, therefore we will also focus on this stream of research.

Acknowledgements: The authors would like to thank Michiel Borgers and Saco Bekius, for providing fruitful insights on their work.

\section{References}

1. O. Akhigbe, D. Amyot, and G. Richards. A framework for a business intelligenceenabled adaptive enterprise architecture. In Conceptual Modeling - 33rd International Conference, ER 2014, Atlanta, GA, USA, October 27-29, 2014. Proceedings, pages 393-406, 2014.

2. D. Amyot et al. Evaluating goal models within the goal-oriented requirement language. Int. J. Intell. Syst., 25:841-877, August 2010. 
3. D. Amyot et al. Towards advanced goal model analysis with jucmnav. In ER Workshops, volume 7518 of LNCS, pages 201-210. Springer, 2012.

4. F. J. Armour, S. H. Kaisler, and S. Y. Liu. A Big-Picture Look at Enterprise Architectures. IT Professional, 1(1):35-42, Jan. 1999.

5. C. Azevedo et al. An Ontology-Based Semantics for the Motivation Extension to ArchiMate. In EDOC'11.

6. G. Chorus, Y. Janse, C. Nellen, S. Hoppenbrouwers, and H. A. Proper. Formalizing Architecture Principles using Object-Role Modelling. Via Nova Architectura, 2007.

7. W. Engelsman and R. Wieringa. Goal-Oriented Requirements Engineering and Enterprise Architecture: Two Case Studies and Some Lessons Learned. In Proceedings of REFSQ, pages 306-320, Berlin, Heidelberg, 2012. Springer-Verlag.

8. C. Fischer, R. Winter, and S. Aier. What is an enterprise architecture principle? - towards a consolidated definition. In Proc. of 9th ACIS, Japan, 2010.

9. S. Ghanavati, D. Amyot, and A. Rifaut. Legal goal-oriented requirement language (Legal-GRL) for modeling regulations. In MiSE @ICSE, 2014.

10. D. Greefhorst and H. Proper. Architecture Principles - The Cornerstones of Enterprise Architecture. EE Series. Springer, Berlin, Germany, 2011.

11. D. Greefhorst, H. A. Proper, and G. Plataniotis. The Dutch State of the Practice of Architecture Principles. Journal of EA, (4):20-25, November 2013.

12. M. K. Haki and C. Legner. New avenues for theoretical contributions in enterprise architecture principles - a literature review. In TEAR/PRET, 2012.

13. M. K. Haki and C. Legner. Enterprise architecture principles in research and practice: Insights from an exploratory analysis. In ECIS, page 204, 2013.

14. A. R. Hevner, S. T. March, J. Park, and S. Ram. Design science in information systems research. MIS Q., 28(1):75-105, Mar. 2004.

15. J. A. P. Hoogervorst. Enterprise architecture: Enabling integration, agility and change. Int. J. Cooperative Inf. Syst., 13(3):213-233, 2004.

16. S. Ingolfo, I. Jureta, A. Siena, A. Perini, and A. Susi. Nomos 3: Legal Compliance of Roles and Requirements. In ER 2014, 2014.

17. ITU-T. Recommendation Z.151 (11/08): User Requirements Notation (URN)Language Definition. http://www.itu.int/rec/T-REC-Z.151/en, 2008.

18. M. Lankhorst et al. Enterprise Architecture at Work: Modelling, Communication and Analysis. Springer, Berlin, Germany, 2005.

19. A. Lindström. On the syntax and semantics of architectural principles. In In Proceedings of (HICSS-39, 4-7 January 2006, Kauai, HI, USA.

20. D. Marosin and S. Ghanavati. Measuring and Managing the Design Restriction of Enterprise Architecture (EA) Principles on EA Models. In Proceedings of 8th RELAW Workshop, 24-28 August 2015, Ottawa, Canada.

21. D. Marosin, S. Ghanavati, and D. van der Linden. A principle-based goal-oriented requirements language (GRL) for enterprise architecture. In Proceedings of the 7th International $i^{*}$ Workshop, Thessaloniki, Greece, 2014.

22. M. Op 't Land and H. A. Proper. Impact of Principles on Enterprise Engineering. In H. Österle, J. Schelp, and R. Winter, editors, The 15th European Conference on Information Systems, pages 1965-1976. University of St. Gallen, St. Gallen, Switzerland, June 2007.

23. G. Plataniotis, S. De Kinderen, Q. Ma, and E. Proper. A conceptual model for compliance checking support of enterprise architecture decisions. In Proceedings of IEEE 17th Conference on Business Informatics (CBI), volume 1, pages 191-198, July 2015. 
24. G. L. Richardson, B. M. Jackson, and G. W. Dickson. A Principles-based Enterprise Architecture: Lessons from Texaco and Star Enterprise. MIS Q., 14(4):385403, 1990.

25. D. Stelzer. Enterprise architecture principles: Literature review and research directions. In A. Dan, F. Gittler, and F. Toumani, editors, ICSOC/ServiceWave Workshops, volume 6275 of LNCS, pages 12-21, 2009.

26. The Open Group. The Open Group - TOGAF Version 9. Van Haren Publishing, Zaltbommel, The Netherlands, 2009.

27. P. van Bommel and et al. Architecture principles - a regulative perspective on enterprise architecture. In EMISA, 2007.

28. P. van Bommel, S. Hoppenbrouwers, H. A. Proper, and T. P. van der Weide. Giving Meaning to Enterprise Architectures: Architecture Principles with ORM and ORC. In OTM Workshops (2), volume 4278 of LNCS, pages 1138-1147. Springer, 2006.

29. M. van Zee, G. Plataniotis, D. Marosin, and D. van der Linden. Formalizing enterprise architecture decision models using integrity constraints. In 16h IEEE Conference on Business Informatics (CBI), May 2014.

30. R. Wieringa. Design science methodology: Principles and practice. In Proceedings of the 32Nd ACM/IEEE International Conference on Software Engineering Volume 2, ICSE '10, pages 493-494, New York, NY, USA, 2010. ACM.

31. M. Wilkinson. Designing an 'adaptive' enterprise architecture. BT Technology Journal, 24(4):81-92, Oct. 2006.

32. R. Winter and S. Aier. How are enterprise architecture design principles used? In EDOCW, pages 314-321. IEEE Computer Society, 2011. 